\title{
HOLOMORPHIC SEPARATION AND THE UNION PROBLEM
}

\author{
GIUSEPPE VIGNA SURIA
}

\begin{abstract}
We give a sufficient condition for an increasing union of holomorphically separated analytic spaces to be holomorphically separated. Furthermore, an example of $\mathbf{J}$. E. Fornaess is investigated in order to show that a union as above is not always holomorphically separated.
\end{abstract}

Introduction. An increasing sequence of analytic spaces is a countable family $\left\{X_{i}\right\}_{i \in N}$ of spaces each contained as an open set in the next. The union problem for Stein spaces asks:

If $\left\{X_{i}\right\}$ is an increasing sequence of Stein spaces, is the union $X=\bigcup_{i \in N} X_{i}$ Stein?

The problem is almost completely solved: precisely, the answer is: yes under either of the following supplementary hypotheses: (a) each $X_{i}$ is Runge in $X_{i+1}$, (b) $X$ is open in a larger Stein manifold, (c) the cohomology group $H^{1}(X, \mathcal{O})$ vanishes; unknown when $X$ is open in a larger Stein space; no, in general, even for manifolds, as shown by a beautiful counterexample due to Fornaess, which seems to avoid falling into cases (a) and (b) by as little as possible.

It is reasonable to ask a similar question for holomorphic separation: precisely

If $\left\{X_{i}\right\}$ is an increasing sequence of holomorphically separated spaces is their union $X$ still holomorphically separated?

Mutatis mutandis it is trivial to see that the answer is yes again in case (a), it is meaningless in case (b), while as for (c), the vanishing of cohomology does not seem to be the right thing to compare with holomorphic separation.

Moreover it is shown here that the counterexample of Fornaess also provides a negative answer to the general union problem for holomorphically separated spaces or manifolds.

Proposition. Let $\left\{X_{i}\right\}_{i \in N}$ be an increasing sequence of holomorphically separated spaces, and suppose that each $X_{i}$ is Runge in $X_{i+1}$. Then $X=\bigcup_{i \in N} X_{i}$ is holomorphically separated.

Proof. Trivial: We know that, since $X_{i}$ is Runge in $X_{i+1} \forall i$, every $X_{i}$ is Runge in $X$ [AG, Proposition 20], i.e. for every compact $K \subseteq X_{i}, f \in \mathcal{O}\left(X_{i}\right)$ and $\varepsilon>0$ there is a $g \in \mathcal{O}(X)$ s.t. $\sup _{K}|f-g|<\varepsilon$. Take $x \neq y$ in $X$ and choose $i$ so large that

Received by the editors December 20, 1983.

1980 Mathematics Subject Classification. Primary 32E15. 
$x, y \in X_{i}$. Since $X_{i}$ is holomorphically separated there is $f$ in $\mathcal{O}\left(X_{i}\right)$ s.t. $f(x)=0$ and $f(y)=2$; now apply the above with $K=\{x, y\}$ and $\varepsilon=1 ; g$ will separate $x$ from $y$.

The Runge hypothesis is necessary, as shown by a little consideration on Fornaess' counterexample to the union problem for Stein manifolds [F]. I briefly remind the reader how it is constructed.

For every positive integer $n$ take the Stein submanifold of $\mathbf{C}^{3}, X_{n}=\left\{(z, w, \eta) \in \mathbf{C}^{3}\right.$ s.t $\left.w \eta=\prod_{k=1}^{n}(z-1 / k)\right\}$ : the function $\gamma_{n}: X_{n} \rightarrow X_{n+1}$, which sends $(z, w, \eta)$ into $(z, w, \eta(z-1 /(n+1)))$, is analytic injective and open. Therefore $X=\lim _{\rightarrow} X_{n}$ is an analytic manifold of dimension 2. Call $[z, w, \eta]$ the class in $X$ of $(z, w, \eta)$ in $X_{n}$; the projection $\pi: \mathbf{C}^{3} \rightarrow \mathbf{C}^{2}$ sending $(z, w, \eta)$ to $(z, w)$ induces an analytic map $\pi$ : $X \rightarrow \mathbf{C}^{2}$, which, restricted to $X-\{w=0\}=:\{[z, w, \eta]$ s.t. $w \neq 0\}$, establishes an analytic isomorphism onto $\mathbf{C} \times \mathbf{C}^{*}=\left\{(z, w) \in \mathbf{C}^{2}\right.$ s.t. $\left.w \neq 0\right\}$, while $\pi^{-1}(z, 0)$ is empty if $z \neq 1 / n$ and $\pi^{-1}(1 / n, 0) \cong \mathbf{C}$, for $n=1,2,3, \ldots$.

I claim that $\pi^{*}: \mathcal{O}\left(\mathbf{C}^{2}\right) \rightarrow \mathcal{O}(X)$, given by $\pi^{*}(f)=f \circ \pi \forall f \in \mathcal{O}\left(\mathbf{C}^{2}\right)$, is an isomorphism. This would imply that every $h \in \mathcal{O}(X)$ is constant on $\pi^{-1}(1 / n, 0), \forall n$, and thus the points above $(1 / n, 0)$ are not separated by any global holomorphic function on $X$.

To prove the claim, i.e. that $\mathcal{O}(X) \subseteq \pi^{*}\left(\mathcal{O}\left(\mathbf{C}^{2}\right)\right)$ (the rest is trivial), take any $h \in \mathcal{O}(X)$ and restrict it to $X-\{w=0\}$; since here $\pi$ is an isomorphism there exists a $g \in \mathcal{O}\left(\mathbf{C} \times \mathbf{C}^{*}\right)$ s.t. $h[z, w, \eta]=g(z, w) \forall[z, w, \eta], w \neq 0$.

The crucial remark is that such a $g$ may be written uniquely as a convergent Laurent series of the form $g(z, w)=\sum_{i=-\infty}^{\infty} g_{i}(z) w^{i}$, where $g_{i} \in \mathcal{O}(\mathbf{C}) \forall i$, and such a series extends to $\mathbf{C}^{2}$ precisely when $g_{i}=0$ for $i<0$; it is precisely our case, in fact, $\forall n$,

$$
\begin{aligned}
\lim _{k \rightarrow \infty} \sum_{i=-\infty}^{\infty} g_{i}\left(\frac{1}{n}\right)\left(\frac{1}{k}\right)^{i} & =\lim _{k \rightarrow \infty} g\left(\frac{1}{n}, \frac{1}{k}\right)=\lim _{k \rightarrow \infty} g \circ \pi\left(\left[\frac{1}{n}, \frac{1}{k}, 0\right]\right) \\
& =\lim _{k \rightarrow \infty} h\left(\left[\frac{1}{n}, \frac{1}{k}, 0\right]\right)=h\left(\left[\frac{1}{n}, 0,0\right]\right)<\infty .
\end{aligned}
$$

But this means that $g_{i}(1 / n)=0 \forall i<0, \forall n$; by the uniqueness of the analytic extension in one variable we get $g_{i}=0$ for $i<0$. Therefore $g$ is actually a function on $\mathbf{C}^{2}$ and the claim is proved.

REMARK. Fornaess proves that $X$ is not Stein by showing that holomorphic convexity fails: this can be seen very neatly after the above claim. In fact if $K$ is a compact of $X$, clearly $\hat{K}_{X}=\pi^{-1}\left(\widehat{\pi(K)}_{\mathbf{C}^{2}}\right)$, so if we take $K=\pi^{-1}(\{|z| \leqslant 1,|w|=1\})$, then $\overline{\pi(K)} \mathrm{C}^{2}$ is the unit polydisc by the maximum principle, so $\hat{K}_{X}$ contains the discrete sequence $\left[\frac{1}{k}, \frac{1}{k}, 0\right], k=1,2, \ldots$, and so is not compact.

Holomorphic separation is requested for Stein spaces, but the psychologically striking feature of Steinness is holomorphic convexity: in this respect, Fornaess' example comes out a little impoverished: it would surely be interesting to see a proof or a counterexample to the following:

If $\left\{X_{i}\right\}$ is an increasing sequence of Stein spaces (manifolds) with $X=\bigcup X_{i}$ holomorphically separated, is $X$ Stein? 


\section{REFERENCES}

[AG] A. Andreotti and H. Grauert, Théorèmes de finitude pour la cohomologie des espaces complexes, Bull. Soc. Math. France 90 (1962), 193-259.

[F] J. E. Fornaess, 2-dimensional counterexamples to generalizations of the Levi problem. Math. Ann. 230 (1977), 169-173.

Istituto di Matematica, Facolta di Scienze, Universita di Trento, Povo 38050, Trento, Italy 